This copy is for your personal, non-commercial use only.

If you wish to distribute this article to others, you can order high-quality copies for your colleagues, clients, or customers by clicking here.

Permission to republish or repurpose articles or portions of articles can be obtained by following the guidelines here.

The following resources related to this article are available online at www.sciencemag.org (this information is current as of September 19, 2013 ):

Updated information and services, including high-resolution figures, can be found in the online version of this article at:

http://www.sciencemag.org/content/341/6151/1219.full.html

Supporting Online Material can be found at:

http://www.sciencemag.org/content/suppl/2013/08/14/science.1240889.DC1.html

A list of selected additional articles on the Science Web sites related to this article can be found at:

http://www.sciencemag.org/content/341/6151/1219.full.html\#related

This article cites 24 articles, 1 of which can be accessed free:

http://www.sciencemag.org/content/341/6151/1219.full.html\#ref-list-1

This article has been cited by 1 articles hosted by HighWire Press; see:

http://www.sciencemag.org/content/341/6151/1219.full.html\#related-urls 


\section{Reversibly Assembled Cellular Composite Materials}

\author{
Kenneth C. Cheung* and Neil Gershenfeld
}

We introduce composite materials made by reversibly assembling a three-dimensional lattice of mass-produced carbon fiber-reinforced polymer composite parts with integrated mechanical interlocking connections. The resulting cellular composite materials can respond as an elastic solid with an extremely large measured modulus for an ultralight material (12.3 megapascals at a density of 7.2 milligrams per cubic centimeter). These materials offer a hierarchical decomposition in modeling, with bulk properties that can be predicted from component measurements and deformation modes that can be determined by the placement of part types. Because site locations are locally constrained, structures can be produced in a relative assembly process that merges desirable features of fiber composites, cellular materials, and additive manufacturing.

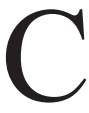
arbon-fiber-reinforced composite materials can improve efficiency in engineered systems (for example, airframes) by reducing structural weight for given strength and stiffness requirements (1), but challenges with manufacturing and certification have slowed their adoption. High-performance composite components are conventionally constructed with many continuous fibers that span the shape of a component and are embedded in a resin matrix that is either pre-impregnated or subsequently infused (2). Parts produced in this way typically require custom tooling to form them, pressurization for consolidation, and heat for matrix curing. Each of these processes multiplies the time, cost, and inflexibility of design, production, and certification. Joint systems between different parts add further complexity and structural vulnerabilities (2).

Center for Bits and Atoms, Massachusetts Institute of Technology, Cambridge, MA 02139, USA.

*Corresponding author. E-mail: kccheung@mit.edu
The approach that we developed uses many small identical parts as regular building blocks. The parts integrate unidirectional fiber composite beams and looped fiber load-bearing holes that are reversibly linked, like chains, to form volumefilling lattice structures. These parts can be massproduced and then assembled to fill arbitrary structural shapes, with a resolution prescribed by the part scale, which is chosen to match the variability of the boundary stress encountered in an application. Each type of identical part can be individually qualified, and as a cellular material, the periodic nature of their assemblies simplifies the analysis and prediction of their behavior.

Fiber composites have been used to construct truss cores $(3,4)$ and structural frames $(5,6)$, with bonded assembly of substructures or continuous winding of fibers over a structure. Examples of such truss cores have been reported with continuous two-dimensional (2D) geometric symmetry and nearly ideal but highly anisotropic specific modulus scaling (4). Three-dimensional (3D) opencell lattice materials occur in natural and engineered systems $(7,8)$, spanning many length scales $(9,10)$, with mechanical properties that scale with relative density according to the geometry of the cellular structure (7). Their response is characterized as displaying either stretch-dominated or transverse beam bending-dominated microstructural behavior, based on periodic mechanical models $(7,11)$. For the Young's modulus $E$, ideal stretch-dominated scaling with density $\rho$ follows a proportional law, $E \propto \rho(12)$; common stochastic foams follow a quadratic law, $E \propto \rho^{2}$ (7), associated with transverse beam bending-dominated behavior; and at ultralight densities, a further reduced cubic scaling law, $E \propto \rho^{3}$, is commonly observed, such as with aerogels and aerogel composites $(7,11,13,14)$.

The dependence of this scaling performance on geometry is seen in nonstochastic lattice-based materials that have nearly ideal $E \propto \rho$ scaling, with high coordination numbers (node connectedness) relative to stochastic foams $(15,16)$. These structures have been implemented thus far only in relatively dense engineered materials. For the ultralight regime [below $10 \mathrm{mg} / \mathrm{cm}^{3}$ (7)], the $E \propto$ $\rho^{2}$ scaling seen in denser stochastic cellular materials has recently been reported for electroplated tubular nickel microlattices (17), as well as carbon-based open-cell stochastic foams, including carbon microtube aerographite (18), and graphene cork (19). Reversibly assembled cellular composites extend stretch-dominated latticebased materials to the ultralight regime, with 3D symmetry derived from the linked geometry. The high-performance characteristics of these new materials depend on the framework rigidity of the nonstochastic lattice geometry with high coordination number, the slenderness that can be achieved in buckling-limited fiber composite strut members, and the scaling of the density cost of reversible mechanical connections.
Fig. 1. Structural components. (A) Assembly of a "cuboct" lattice of vertex-connected octahedrons from planar components linked by shear clips [see fig. S5 (20) for additional detail of the spatial arrangement of parts]. (B) Carbon fiber-reinforced polymer composite cuboct lattice. (C) Reversible joints of components in the cuboct lattice. (D) Micrograph showing fiber orientation around connection holes and along strut members. (E) Slicing components from wound/oriented-fiber stock rather than conventionally cutting quasi-isotropic laminate.
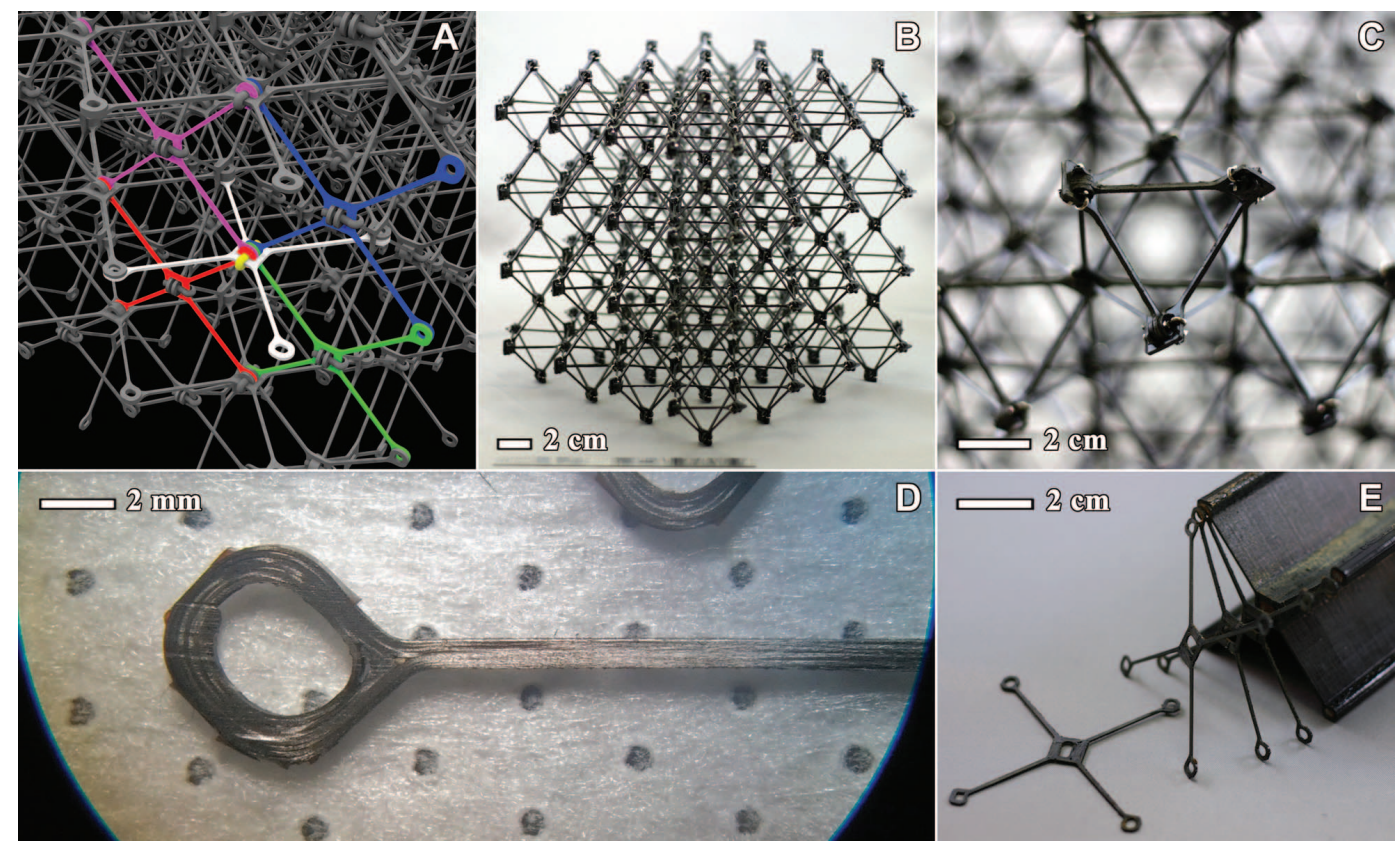
We designed the connections to be stiffer and stronger than the strut members, so that the response of these structures is governed by the struts [see supplementary materials (20)]. Dimensional scaling methods (7), extended to include the connections (20), show that the mass density cost of such robust connections - which scale with the cross-sectional area of strut members - is low for ultralight materials, where strut member slenderness dominates mass density scaling. We define the relative density $\left(\rho / \rho_{s}\right)$ of these materials as the sum of the relative density contribution of the strut members $\left(\rho_{\mathrm{m}} / \rho_{s}\right)$ and the relative density contribution of the connections $\left(\rho_{\mathrm{c}} / \rho_{s}\right)$. The strut members have a thickness $t$ and length $l$. The connections were designed to transfer forces through load-bearing surface contacts, and this constraint required that the characteristic dimensions of the connections must scale with the cross-sectional area of the attached strut members, $t^{2}$, because this dimension determines the maximum possible stress transferrable through the joint.

These definitions give a cubic scaling relation between the relative mass contribution of the joints and the thickness-to-length ratio of the strut members $\left[\rho_{\mathrm{c}} / \rho_{s} \propto C_{\mathrm{c}}(t / l)^{3}\right.$, where $C_{\mathrm{c}}$ is the connection contribution constant determined by the lattice geometry]. We find that the relative density contribution of the strut members is governed by quadratic scaling with the thickness-to-length ratio of the strut members $\left[\rho_{\mathrm{m}} / \rho_{s} \propto C_{\mathrm{m}}(t / l)^{2}\right]$, which agrees with the literature on classical cellular materials $(7,11)$. Mechanical properties (such as modulus and strength) scale with overall relative density (7), which in turn scales primarily with the strut and not the connection, because we consider only open-cell lattices with slender strut members $[t / l<0.1(7)]$, and because the geometric constants $C_{\mathrm{c}}$ and $C_{\mathrm{m}}$ are of the same order of magnitude $\left[\rho / \rho_{s} \propto C_{\mathrm{c}}(t / l)^{3}+C_{\mathrm{m}}(t / l)^{2}\right.$; see supplementary materials for expanded derivation and tables for geometric constants (20)]. The density cost of the mechanical joints decreases with increasing strut member slenderness (decreasing $t / l$ ) and decreasing relative density.

The lattice geometry that we introduce here is a "cuboct" cubic lattice of vertex-connected octahedrons, similar to the perovskite mineral structure. It was chosen as a minimally spatially dense lattice with a regular polyhedral unit cell that satisfies Maxwell's rigidity criterion (21), and has a coordination number $z$ of eight. Comparing lattice geometries, the dependence of the relative density on the coordination number is small relative to the dependence on the strut member slenderness (7), even when including the contribution of connections. As shown in Fig. 1, the reinforcing fibers were wound around the connection holes to optimize their load-bearing capacity while coupling them to strut members, which themselves retain uniaxial fiber orientation. The cross-shaped parts tiled space to form the cuboct lattice structure with each part contributing four conjoined strut members to one locally central node and one strut member to four peripheral nodes in the lattice. After all parts at each connection were in place, a shear clip was inserted through the four coincident connection holes (Fig. 1A).

The assembled material responded as an elastic solid in both tension and compression, with a linear regime followed by a nonlinear superelastic deformation mode (Fig. 2A). The residual hysteresis was primarily caused by the fixture [fig. S1 (20)]. The modulus data in Fig. 3 were calculated from the linear elastic regime
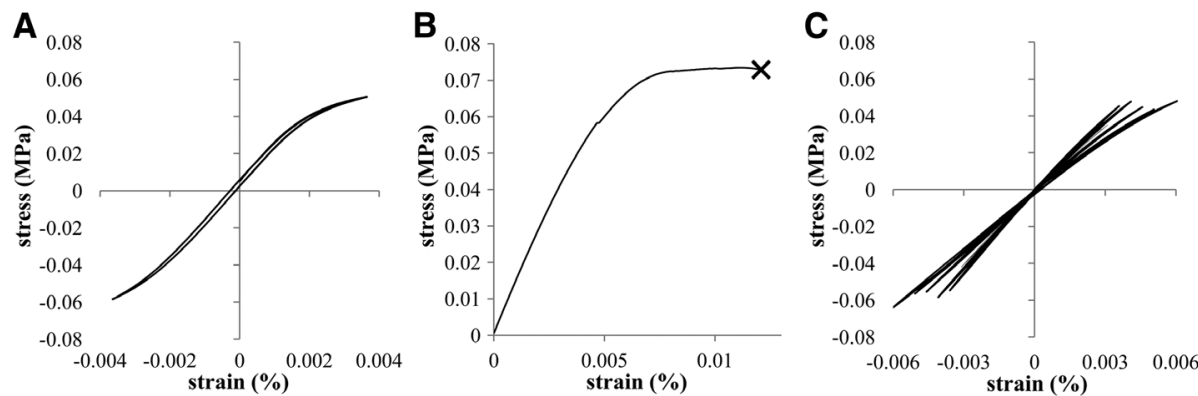

Fig. 2. Mechanical response. (A) Load cycling under compression and tension, showing a linear elastic response followed by a nonlinear superelastic response due to coordinated buckling limage and simulation shown in fig. S1 (20)]. (B) Testing to failure, defined by an abrupt decrease in stress by at least $10 \%$, with failure by microstructural beam bending failures and associated $\sigma_{\max } \propto \rho^{1.5}$ scaling (7). (C) Elastic load cycling after failure, showing incremental softening.

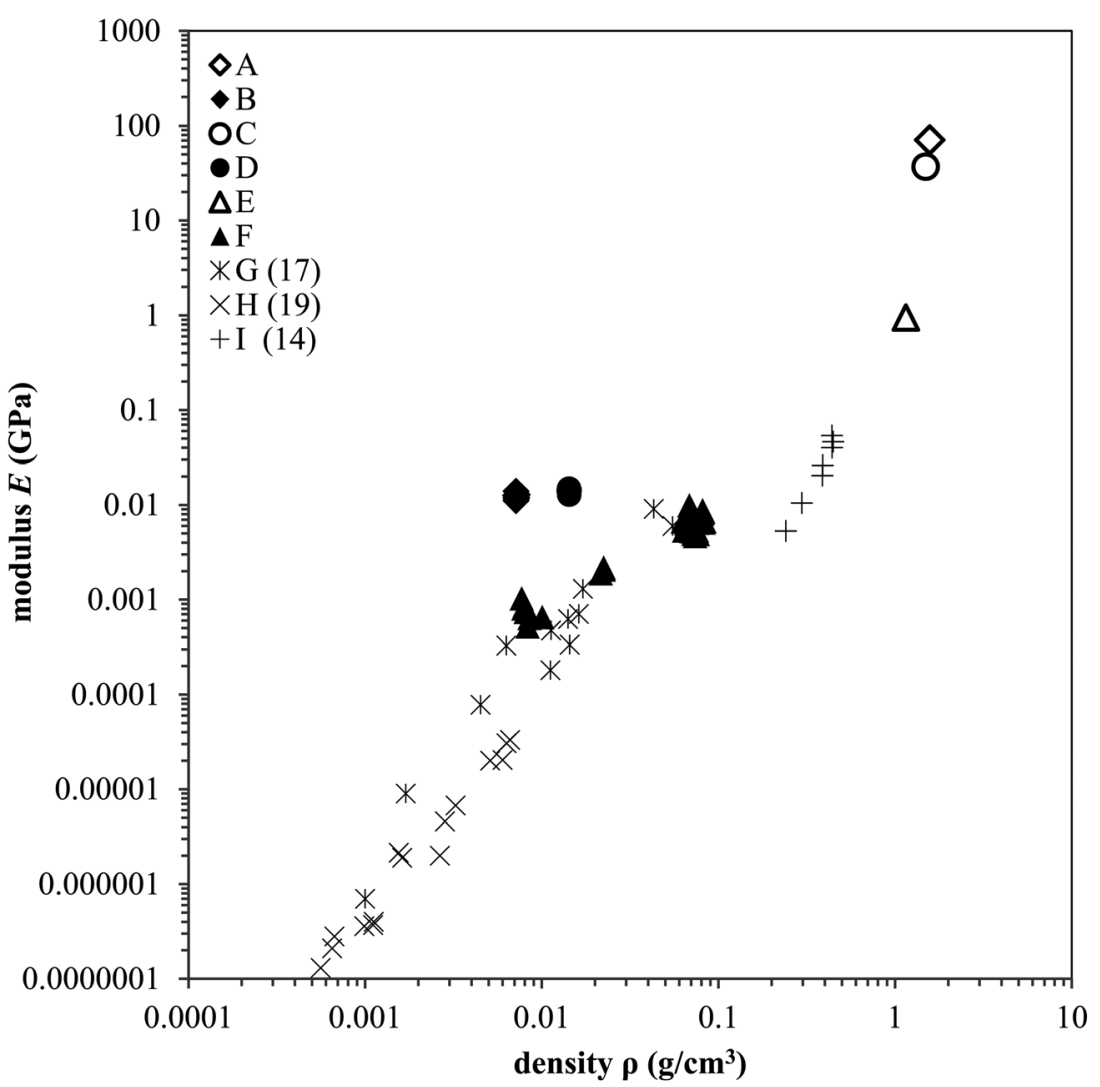

Fig. 3. Materials comparison. Young's modulus versus density for (A) unidirectional carbon fiberreinforced polymer composite solid (along fiber axis), (B) reversibly assembled cellular composite material with wound/oriented-fiber parts, (C) conventional quasi-isotropic carbon fiber-reinforced polymer composite solid (laminate measured along quasi-isotropic plane), (D) reversibly assembled cellular composite material with parts conventionally cut from quasi-isotropic laminate, (E) acrylic solid, (F) acrylic cuboct lattice-based cellular material, (G) metallic micro-lattice (17), (H) graphene cork (19), and (I) silica aerogel composite (14). Data tables are provided in the supplementary materials (20). 
by the simple periodic models (22), and by natural distributions of morphological defects (23).

The modulus of the reversibly assembled cellular composite structure was more than an order of magnitude greater than that of reported ultralight materials, $12.3 \mathrm{MPa}$ at a density of $7.2 \mathrm{mg} / \mathrm{cm}^{3}$. We attribute the nonlinear elastic behavior to a multi-axial elastic instability of the lattice, a complex coordinated elastic buckling of the strut members. The resulting geometry is similar to a Jahn-Teller distortion of an octahedral complex with respect to orientation about the octahedral centers. Elastic folding or pleating across a lattice structure has been described as a coordinated antisymmetric twisting stress response in 2D lattices (24), and plastic deformations have been seen in auxetic lattice materials (7). We observed similar responses in three dimensions [see movie S1, showing corresponding affine rotations of terminal end nodes during loading (20)].

Figure 2B shows the plateau before failure, as defined by an abrupt decrease in stress by at least $10 \%$. In Fig. 2C, repeated cycling in the elastic limit is shown after failure events. Unlike conventional composites, which fail catastrophically, these structures can fail incrementally because of the nonlinear deformation phase and the multiplicity of joints and links. These results are supported by finite-element simulations (ANSYS software) with finely meshed rigid body models. In addition to convergence to the observed coordinated buckling mode [fig. S3 (20)], these simulations also accurately predict the relative strength scaling observed in load test experiments. These results are consistent with the observation that open-cell lattice materials fail through microstructural bending failures in strut members, with $\sigma_{\max } \propto \rho^{1.5}$ (7). The simulations also suggest that the coordinated buckling phenomenon, as well as the modulus measurements, is not dominated by edge effects (25), with minimal influence on overall results beyond characteristic dimensions exceeding several units [fig. S4 (20)].

The ability to tune anisotropic mechanical properties of fiber composite materials is considered to be an important capability (26). The discrete construction of reversibly assembled cellular composites introduces a new design degree of freedom that allows global functional properties to be determined by the local placement of compatible heterogeneous components. Figure 4 shows three structures with the same lattice geometry, differing only in the relative locations of a more-rigid and less-rigid component, which under identical constraints and loading will axially compress or buckle with prescribed modes. In this fashion, heterogeneous elements can be incorporated into multifunctional structures with properties determined by their relative placement, so that mechanisms can be built out of rather than into the regular components. Because the individual parts are literally finite elements, there is a natural hierarchical decomposition in modeling between that of the part types and their combination in a structure.

Reversibly assembled cellular composites can be considered to be a "digital" material (27): A discrete set of parts is linked with a discrete set of relative positions and orientations. These attributes allow an assembler to place them using only local information rather than global positioning, and allow placement errors to be detected and corrected. These attributes are familiar in nanoassembly (28) and are used here on macroscopic scales. These materials combine the size and strength of composites with the low density of cellular materials and the convenience of additive manufacturing. The need for custom tooling is eliminated, because parts can be incrementally added to a structure. Their construction, modification, repair, and reuse can all employ the same reversible linking process. Heterogeneous elements can be incorporated in structures with functions determined by their rel-

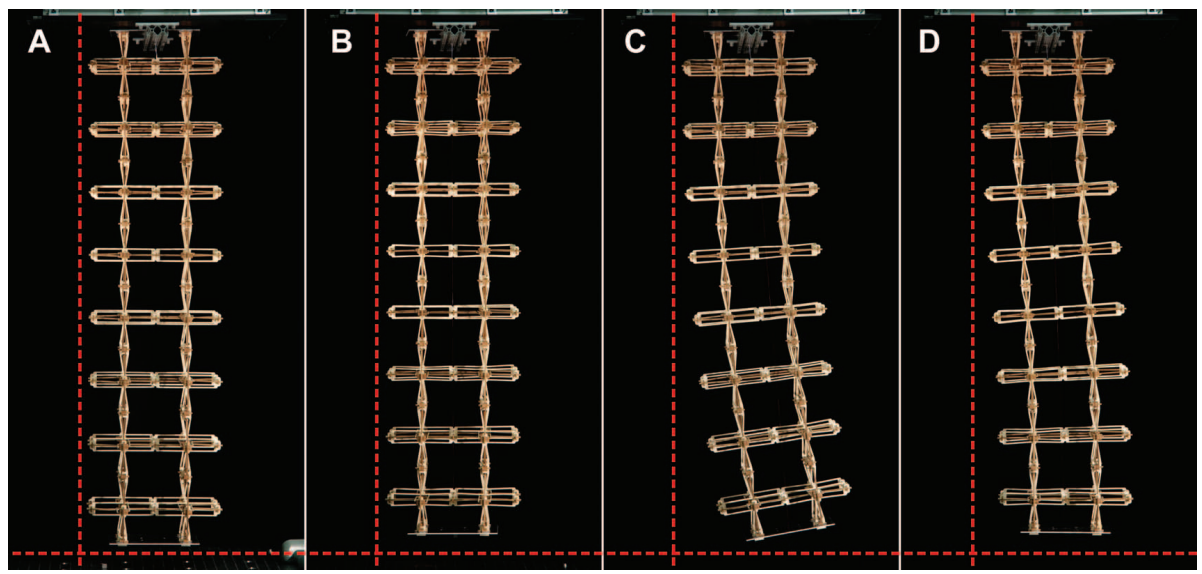

Fig. 4. Deformations designed by the relative placement of rigid and flexural components. Photographs (A to D) are shown, with initial conditions (A) and red datum lines for comparison. Using identical sets of these two part types, the structures shown ( $B$ to $D$ ) differ only by the spatial arrangement of these parts. Under identical constraints and loading conditions (provided by a cable running through the center of the structure), we see pure axial compression in one example (B), simple Euler buckling (biased to occur in one direction) in another example (C), and complex buckling in the third example (D). Part placement diagrams and simulations for the differing structures are shown in fig. S6 (20). ative placement. Exact assembly of discrete cellular composites offers new properties and performance not available with the analog alternatives of continuously depositing or removing material.

\section{References and Notes}

1. A. Baker, S. Dutton, D. W. Kelly, Composite Materials for Aircraft Structures (American Institute of Aeronautics and Astronautics, Reston, VA, ed. 2, 2004)

2. S. Mazumdar, Composites Manufacturing: Materials, Product, and Process Engineering (CRC Press, New York, 2002).

3. J. Xiong, L. Ma, L. Wu, B. Wang, A. Vaziri, Compos. Struct. 92, 2695-2702 (2010).

4. K. Finnegan, G. Kooistra, H. N. Wadley, V. S. Deshpande, Int. J. Mater. Res 98, 1264-1272 (2007).

5. V. V. Vasiliev, A. F. Razin, Compos. Struct. 76, 182-189 (2006).

6. A. D. Reddy, R. Valisetty, L. W. Rehfield, J. Aircr. 22, 249-255 (1985).

7. L. G. Gibson, M. F. Ashby, Cellular Solids, Structures and Properties (Cambridge Univ. Press, Cambridge, ed. 2, 1997).

8. R. C. Thomson, M. J. Yaszemski, ]. M. Powers, A. G. Mikos, J. Biomater. Sci. Polym. Ed. 7, 23-38 (1996)

9. D. A. Head, A. J. Levine, F. C. MacKintosh, Phys. Rev. Lett. 91, 108102 (2003).

10. J. Banhart, Prog. Mater. Sci. 46, 559-632 (2001)

11. V. S. Deshpande, M. F. Ashby, N. A. Fleck, Acta Mater. 49, 1035-1040 (2001).

12. M. F. Ashby, Acta Metall. 37, 1273-1293 (1989).

13. H. Ma, A. P. Roberts, J. Prévost, R. Jullien, G. W. Scherer, J. Non-Cryst. Solids 277, 127-141 (2000).

14. N. Leventis, C. Sotiriou-Leventis, G. Zhang, A. M. Rawashdeh, Nano Lett. 2, 957-960 (2002)

15. V. S. Deshpande, N. A. Fleck, M. F. Ashby, J. Mech. Phys. Solids 49, 1747-1769 (2001).

16. D. J. Sypeck, H. N. Wadley, J. Mater. Res. 16, 890-897 (2001).

17. T. A. Schaedler et al., Science 334, 962-965 (2011).

18. M. Mecklenburg et al., Adv. Mater. 24, 3486-3490 (2012).

19. L. Qiu, J. Z. Liu, S. L. Chang, Y. Wu, D. Li, Nat. Commun. 3, 1241 (2012)

20. Materials and methods are available as supplementary materials on Science Online.

21. J. C. Maxwell, Philos. Mag. 4, 294-299 (1864)

22. A. P. Roberts, E. J. Garboczi, J. Mech. Phys. Solids $\mathbf{5 0}$ 33-55 (2002).

23. V. S. Deshpande, N. A. Fleck, J. Mech. Phys. Solids $\mathbf{4 8}$ 1253-1283 (2000)

24. K. Bertoldi, P. M. Reis, S. Willshaw, T. Mullin, Adv. Mater. 22, 361-366 (2010).

25. E. W. Andrews, G. Gioux, P. Onck, L. J. Gibson, Int. J. Mech. Sci. 43, 701-713 (2001)

26. D. Hull, T. W. Clyne, An Introduction to Composite Materials, D. R. Clark, S. Suresh, I. M. Ward, Eds., Cambridge Solid State Science Series (Cambridge Univ. Press, Cambridge, ed. 2, 1996).

27. G. Popescu, N. Gershenfeld, T. Marhale, "Digital materials for digital printing," International Conference on Digital Fabrication Technologies, Denver, CO (2006)

28. K. Fujibayashi, D. Y. Zhang, E. Winfree, S. Murata, Nat. Comput. 8, 589-612 (2009).

Acknowledgments: This work was supported by Defense Advanced Research Projects Agency's Maximum Mobility and Manipulation program (W911NF-11-1-0096) and the Center for Bits and Atoms sponsors. We acknowledge the contribution of Spirit AeroSystems on composite development, as well as J. H. Kim and S. Hovsepian for research assistance. Patent applications have been filed for the design and manufacturing of reversibly assembled cellular composite materials.

\section{Supplementary Materials}

www.sciencemag.org/cgi/content/full/science.1240889/DC1 Materials and Methods

Figs. S1 to S8

Tables S1 and S2

References (29-32)

Movie $\mathrm{S} 1$

22 May 2013; accepted 30 July 2013

Published online 15 August 2013;

10.1126/science.1240889 